\title{
Estudio Morfométrico de las Suturas Palatinas Humanas en Recién Nacidos, Infantes y Niños con Fines de Tratamiento por Distracción Osteogénica
}

\author{
Morphometrical Study of Human Palatine Sutures in Newborns, Infants \\ and Children for Distraction Osteogenesis Treatment Purposes
}

Cantín, M.,"**; Olate, S.,"**; Fonsea, C.***; Inzunza, O. .**; Contreras, F.*** \& Salgado, G.***

CANTIN, M.; OLATE, S.; FONSEA, C.; INZUNZA, O.; CONTRERAS, F. \& SALGADO, G. Estudio morfométrico de las suturas palatinas humanas en recién nacidos, infantes y niños con fines de tratamiento por distracción osteogénica. Int. J. Morphol., 31(3):11301136, 2013.

RESUMEN: El conocimiento estructural y dimensional de las suturas palatinas es necesario para el tratamiento temprano de deficiencias transversales y longitudinales por técnicas de expansión o distracción osteogénica (DO). El objetivo fue estudiar el estado de las suturas palatinas y los cambios cambios dimensionales y morfológicos desde el nacimiento a la niñez. Fueron analizados 41 paladares óseos de ambos sexos entre 0 y 13 años, agrupándolos en recién nacidos (RN/n=17), infantes (IN/n=12) y niños (NI/n=12). Se fotografió cada paladar y se marcaron puntos craneométricos para determinar las dimensiones longitudinales y transversales de las suturas palatinas mediana premaxilar (SPPMX), mediana anterior (SPMA), posterior (SPMP), transversa anterior (SPTA) y transversa posterior (SPTP). Además se evaluó el estado sutural y dimorfismo sexual. Los resultados se sometieron a las pruebas OneWay-ANOVA, Bonferroni y ttest. Las suturas SPMA, SPMP y SPTP no presentaron sinostosis. La SPPMX se observo parcialmente sinostosada en 5,9\% de RN y $16,7 \%$ de NI, y la SPTA en $8,3 \%$ de IN y $41,7 \%$ de NI. Al comparar la longitud sutural en los tres grupos, las dimensiones longitudinales estadísticamente significativas en todos los grupos $(\mathrm{p}<0,001)$. No hubieron diferencias en las suturas transversales entre IN y NI ( $\mathrm{p}=0,32)$. Se observó dimorfismo en IN entre las SPMA y SPTP. El sistema sutural palatino juega un rol importante durante el crecimiento por su disposición sagital y transversal, permitiendo un crecimiento bidireccional del paladar. El crecimiento longitudinal es constante, mientras que el transversal muestra un peak hasta los 2 primeros años de vida y luego disminuye, sugiriendo la existencia de diferentes potenciales de crecimiento. Estas observaciones podrían explicar la alta prevalencia de alteraciones de crecimiento transversal en los niños. Estos datos son relevantes para el tratamiento oportuno por expansión o DO en casos de compresión del paladar, incompetencia velofaríngea o fisuras palatinas.

PALABRAS ClAVE: Suturas palatinas; Paladar duro; Paladar óseo; Crecimiento; Distracción ósea.

\section{INTRODUCCIÓN}

En el paladar óseo, los procesos palatinos del hueso maxilar y las láminas horizontales del hueso palatino se encuentran interconectadas por las suturas palatinas, la que se organizan en dos sistemas, sagital y transversal, y permiten el crecimiento palatino en sentido antero-posterior y medio-lateral respectivamente (Silau et al., 1994). El sistema sutural palatino humano comprende en jóvenes y adultos las suturas palatinas mediana anterior (SPMA), mediana posterior (SPMP) y transversa (SPT); también encontramos una sutura premaxilar (SPPMX) (Lathan, 1971), y la sutura palatina transversa anterior (SPTA), pero ambas se sinostosan tempranamente.
El conocimiento de la disposición estructural y desarrollo de las suturas palatinas es útil para entender la etiolopatogénesis de malformaciones como el Torus palatinus, así como para tratar deficiencias transversales y longitudinales (compresiones) del paladar en niños, jóvenes y adultos (Stuart \& Wiltshire, 2003; N'Guyen et al., 2007).

La distracción osteogénica (DO) es un tratamiento estándar de expansión esquelética craneofacial. Esta técnica se ha utilizado ampliamente en maxilar, mandíbula, procesos alveolar y frontoorbitario, así como también en el paladar (Rojvachiranonda et al., 2003). En humanos, la DO ha

* CIMA, Facultad de Odontología, Universidad de la Frontera, Temuco, Chile.

** Centro de Investigación en Ciencias Biomédicas, Universidad Autónoma de Chile, Temuco, Chile.

*** Departamento de Anatomía, Facultad de Medicina, Pontificia Universidad Católica de Chile, Santiago, Chile. 
sido reportada por Taub et al. (2001), quienes lograron cerrar mediante este procedimiento una fístula oronasal de un hombre de 53 años de edad, después de tres intentos de cirugía reconstructiva fallidos debido a terapias de irradiación previa. También se ha descrito la utilización de la DO para ampliar la constricción maxilar a través de la sutura palatina mediana en niños y adolescentes.

Actualmente se utilizan dispositivos de expansión palatina dentosoportados, capaces de generar efectos secundarios desfavorables como la flexión del hueso alveolar, compresión de la membrana periodontal, fenestración de la cortical vestibular del hueso, y el desplazamiento lateral del diente, donde una técnica de DO puede evitar estas complicaciones (Rojvachiranonda et al.). Carls et al. (1997a, b) realizaron con exíto una técnica quirúrgica para tratar la incompetencia velofaríngea mediate la distracción de los huesos del paladar en perros; mientras que Liu et al. (2000) reportaron una serie de estudios experimentales que demuestra el cierre quirúrgico del paladar hendido y el avance simultáneo del maxilar y el paladar. Para poder aplicar estas técnicas en humanos, es necesario conocer la normalidad de las estructuras palatinas y abordar problemas relacionados con los defectos del paladar.

Son escasos los estudios centrados en las suturas palatinas, y principalmente describen sus características en sujetos adultos, con muestras relativamente pequeñas, sin variaciones de edad, por lo que se ha sugerido realizar estudios con una mayor muestra y desde las primeras etapas de vida (Ferrario et al., 2000; Skrzat et al., 2003; Rojvachiranonda et al.; N'Guyen et al.). Rojvachiranonda et al. describieron las características palatinas y suturales en paladares de 3 fetos y 5 recién nacidos ya que se carece de información acerca de las suturas del paladar durante la infancia, concluyendo la necesidad de realizar estudios en el área.

Por lo tanto, es necesario realizar un estudio anatómico adecuado con el fin de tener antecedentes anatómicos normales para el tratamiento de patología del desarrollo que afectan al paladar.

\section{MATERIAL Y MÉTODO}

Se realizó un estudio descriptivo observacional, de corte transversal. Fueron analizados cráneos de ambos sexos, con registro de edad entre 0 y 13 años pertenecientes a la Colección del Museo de Anatomía de la Universidad Federal de la São Paulo (UNIFESP). Se incluyeron cráneos con indemnidad estructural de la zona palatina, y excluyeron los casos que evidenciaran presencia de malformaciones palatinas que pudieran sesgar la obtención de datos. La muestra quedó constituida por 41 cráneos, agrupados en tres categorías según edad en recién nacidos (RN), infantes (IN) y niños (NI) (Tabla I).

Desde cada cráneo, fueron fotografiados los paladares óseos siguiendo el método descrito por Moreira et al. (2006). Sobre las imágenes digitales de los paladares óseos y con el software ScreenScales se determinaron los puntos anatómicos foramen incisivo (fin), estaurion (sr), espina nasal posterior (enp) y foramen palatino mayor (fpm) en cada caso y se realizaron mediciones lineales en ellos. Las longitudes registradas correspondieron a la sutura palatina mediana premaxilar (SPPMX), sutura palatina mediana anterior (SPMA), sutura palatina mediana posterior (SPMP), sutura palatina transversa anterior (SPTA) y sutura palatina transversa posterior (SPTP) (Fig. 1).

Se evaluó el estado de las suturas en cada grupo, registrando si se encontraban presentes, ausentes o parcialmente sinostosadas. Además, se determinó el labio de unión de la SPTP en relación al foramen palatino mayor (anterior, medial o póstero-lateral) para evaluar el posible compromiso del paquete neurovascular.

Las distancias de las cinco suturas palatinas fueron medidas independientemente por dos examinadores y los valores obtenidos para cada parámetro fueron promediados. Las mediciones que presentaron una diferencia interexaminador superior al $10 \%$ fueron repetidas. Una vez tabulados los datos de los distintos grupos, se realizó el análisis estadístico descriptivo e inferencial con el software computacional SigmaPlot $@$ (v12.0), con un intervalo de confianza del $95 \%$. Se analizaron los valores de las cinco suturas palatinas descritas en la muestra, en busca de diferencias estadísticamente significativas entre los tres grupos estudiados aplicando la prueba OneWay ANOVA. También se realizó una comparación pareada de los valores de cada sutura entre los distintos grupos (RN vs. IN, RN vs. NI, e IN vs. NI) en busca de diferencias aplicando la prueba de Bonferroni. Además se realizó una comparación por sexo de los valores dentro de cada grupo aplicando la prueba t de Student. Al comparar los datos, se considero una diferencia significativa un valor $\mathrm{p}<0,05$.

Tabla I. Caracterización de la muestra estudiada por grupo y edad.

\begin{tabular}{lcccc}
\hline Grupo & Edad (años) & Hombr & Mujeres & Total \\
\hline Recién nacido (RN) & $0-0,5$ & 10 & 7 & 17 \\
Infante (IN) & $>0,5-2$ & 4 & 8 & 12 \\
Niño (NI) & $>2-13$ & 7 & 5 & 12 \\
\hline
\end{tabular}




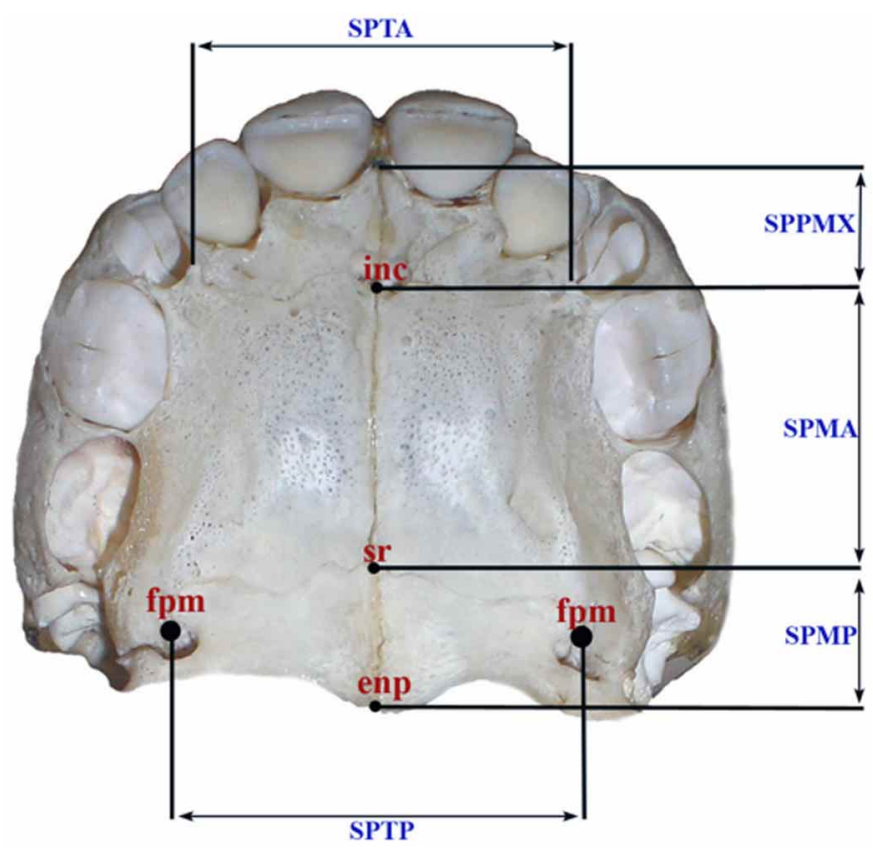

Fig. 1. Puntos anatómicos determinados en del paladar ósea y las longitudes registradas.

\section{RESULTADOS}

En todos los paladares se observaron los cinco grupos suturales. La SPMA, SPMP y SPTP no mostraron signos de sinostosis, mientras que la SPPMX se observo parcialmente sinostosada en el 5,9\% de los RN y $16,7 \%$ de los niños, y la SPTA se mostró parcialmente sinostosada en el 8,3\% de los infantes y en el $41,7 \%$ de los niños.

Las longitudes para cada sutura según grupos etáreos se observan en la Tabla II. Al comparar las medidas de longitud promedio de las suturas palatinas del grupo RN vs. NI, se encontraron diferencias significativas en todos los parámetros estudiados $(\mathrm{p}<0,001)$, mientras que al comparar los grupos $\mathrm{RN}$ vs. IN se encontraron diferencias para la $\operatorname{SPTA}(\mathrm{p}=0,01)$ y SPTP $(\mathrm{p}<0,001)$. En los grupos IN vs. NI, se encontraron diferencias significativas para la SPPMX $(\mathrm{p}=0,01)$, SPMA $(\mathrm{p}=0,004)$ y $\operatorname{SPMP}(\mathrm{p}=0,015)$ (Tabla III).

En el grupo RN al comparar las mediciones entre hombres y mujeres, no se encontraron diferencias significativas $(0,097<\mathrm{p}<0,662)$. La Media obtenida para la SPPMX en hombres fue 6,78 $\pm 1,53 \mathrm{~mm}$ y en mujeres $6,14 \pm 1,35 \mathrm{~mm}(\mathrm{p}=0,223)$, para la SPMA en hombres fue $14,092 \pm 1,753 \mathrm{~mm}$ y en mujeres $13,55 \pm 0,97 \mathrm{~mm}$ $(\mathrm{p}=0,475)$, para la SPMP en hombres fue 4,61 $\pm 1,50 \mathrm{~mm}$ y en mujeres $5,38 \pm 1,94 \mathrm{~mm}(\mathrm{p}=0,37)$, para la SPTA en hombres fue 15,32 $\pm 1,62 \mathrm{~mm}$ y en mujeres $15,68 \pm 1,67$ mm $(\mathrm{p}=0,662)$ y para la SPTP en hombres fue $18,68 \pm 1,39 \mathrm{~mm}$ y en mujeres $19,82 \pm 1,99 \mathrm{~mm}(\mathrm{p}=0,097)$

En el grupo IN se encontraron diferencias significativas entre hombres y mujeres, al comparar las medidas de las SPMA y SPTP ( $\mathrm{p}=0,042$ y $\mathrm{p}=0,04$ respectivamente) (Tabla IV).

En el grupo de NI no se encontraron diferencias significativas para ninguno de los parámetros al comparar hombres y mujeres $(0,343<\mathrm{p}<0,882)$. Para SPPMX en hombres la Media obtenida fue $9,42 \pm 1,57 \mathrm{~mm}$ y en mujeres $10,20 \pm 1,77 \mathrm{~mm}$ ( $\mathrm{p}=0,343$ ), para SPMA en hombres fue 20,51 $\pm 5,19 \mathrm{~mm}$ y para mujeres $20,13 \pm 2,32 \mathrm{~mm}$ $(\mathrm{p}=0,882)$, para la SPMP en hombres fue 8,230 $\pm 7,380$ mm y en mujeres $7,41 \pm 6,61 \mathrm{~mm}(\mathrm{p}=0,876)$, para la SPTA en hombres fue $20,62 \pm 3,82 \mathrm{~mm}$ y en mujeres $19,56 \pm 1,98$ $(\mathrm{p}=0,584)$ y para la SPTP en hombres fue $25,66 \pm 2,94$ $\mathrm{mm}$ y en mujeres $24,30 \pm 3,68 \mathrm{~mm}(\mathrm{p}=0,492)$.

Respecto a la ubicación del labio de unión de la SPTP en relación al FPM, fue posible establecer en RN que el extremo derecho termino en un $41,2 \%$ por anterior, $11,8 \%$ por medial y un $47,1 \%$ posterolateral al foramen, y para el extremo izquierdo un $35,3 \%$ por anterior, un $5,9 \%$ por medial y un $58,8 \%$ posterolateral al foramen. En el caso de IN, el extremo derecho de la

Tabla II. Longitud de las suturas palatinas SPPMX, SPMA, SPMP, SPTA y SPTP en los 41 cráneos según grupos etáreos de recién nacidos, infantes y niños. Se observan los valores de Media, Error Estándar, Máximo y Mínimo para cada caso.

\begin{tabular}{lcrrrrrrrr}
\hline & \multicolumn{3}{c}{ Recién nacid os } & \multicolumn{3}{c}{ Infantes } & \multicolumn{3}{c}{ Niños } \\
\hline & $\begin{array}{c}\text { Media } \pm \text { EE } \\
(\mathbf{m m})\end{array}$ & Max. & Mín. & \multicolumn{1}{c}{$\begin{array}{c}\text { Media } \pm \text { EE } \\
(\mathbf{m m})\end{array}$} & Max. & Mín. & \multicolumn{1}{c}{$\begin{array}{c}\text { Media } \pm \text { EE } \\
(\mathbf{m m})\end{array}$} & Max. & Mín. \\
\hline SPPMX & $6,50 \pm 1,41$ & 8,08 & 3,10 & $7,85 \pm 1,40$ & 9,86 & 4,81 & $9,74 \pm 1,63$ & 11,80 & 7,44 \\
SPMA & $13,87 \pm 1,47$ & 17,41 & 12,50 & $16,35 \pm 2,74$ & 21,35 & 12,86 & $20,36 \pm 4,09$ & 27,66 & 13,05 \\
SPMP & $4,93 \pm 1,68$ & 8,12 & 1,52 & $6,39 \pm 1,68$ & 10,55 & 3,56 & $8,79 \pm 2,55$ & 15,10 & 5,81 \\
SPTA & $15,46 \pm 1,60$ & 18,33 & 13,19 & $18,48 \pm 2,96$ & 25,00 & 14,87 & $20,18 \pm 3,11$ & 24,62 & 12,80 \\
SPTP & $19,15 \pm 1,70$ & 23,00 & 16,67 & $23,05 \pm 2,83$ & 28,41 & 19,34 & $25,09 \pm 3,18$ & 32,25 & 18,87 \\
\hline
\end{tabular}


sutura se ubico un $8,3 \%$ por anterior y en $91,7 \%$ posterolateral al foramen, y el extremo izquierdo se ubicó en un $16,7 \%$ por anterior y en un $83,3 \%$ posterolateral al foramen. En niños el extremo derecho de la sutura se ubicó en un $41 \%$ de los casos por anterior y $59 \%$ posterolateral al foramen, y el extremo izquierdo se ubico en un $33,3 \%$ por anterior, en $16,7 \%$ por medial y en $50 \%$ de los casos posterolateral al FPM.

Tabla III. Diferencias estadísticas de las suturas palatinas entre los diferentes grupos analizados.

\begin{tabular}{lcccccc}
\hline & \multicolumn{2}{c}{ RN vs. NI } & \multicolumn{2}{c}{ RN vs. IN } & \multicolumn{2}{c}{ IN vs,.NI } \\
\hline & $\mathbf{p}<\mathbf{0 , 0 5}$ & Valor $\mathbf{p}$ & $\mathbf{p}<\mathbf{0 , 0 5}$ & Valor $\mathbf{P}$ & $\mathbf{p}<\mathbf{0 , 0 5}$ & Valor $\mathbf{p}$ \\
\hline SPPMX & SI & $<0,001 * * *$ & NO & 0,059 & SI & $0,01 * *$ \\
SPMA & SI & $<0,001 * * *$ & NO & 0,073 & SI & $0,004^{* *}$ \\
SPMP & SI & $<0,001 * * *$ & NO & 0,171 & SI & $0,015^{*}$ \\
SPTA & SI & $<0,001 * * *$ & SI & $0,01 * *$ & NO & 0,325 \\
SPTP & SI & $<0,001 * * *$ & SI & $<0,001 * * *$ & NO & 0,17 \\
$*$ Valor $p<0.05, * * *$ Valor $p<0.001$. & & & &
\end{tabular}

Tabla IV. Diferencias de las suturas palatinas según sexo en infantes.

\begin{tabular}{lccccccc}
\hline & \multicolumn{3}{c}{ Infantes hombres } & \multicolumn{5}{c}{ Infantes mujeres } & \\
\cline { 2 - 6 } & Media \pm EE $(\mathbf{m m})$ & Máx, & Mín, & Media \pm EE $(\mathbf{m m})$ & Máx, & Mín, & Valor p \\
\hline SPPMX & $8,39 \pm 0,32$ & 8,77 & 8,11 & $7,59 \pm 1,67$ & 9,86 & 4,80 & 0,46 \\
SPMA & $18,55 \pm 2,90$ & 21,35 & 14,63 & $15,25 \pm 2,01$ & 18,28 & 12,86 & $0,042^{*}$ \\
SPMP & $7,28 \pm 2,18$ & 10,55 & 5,92 & $5,95 \pm 1,31$ & 8,11 & 3,56 & 0,21 \\
SPTA & $19,69 \pm 4,37$ & 25,00 & 15,85 & $17,87 \pm 2,09$ & 20,43 & 14,87 & 0,57 \\
SPTP & $25,34 \pm 2,56$ & 28,41 & 22,50 & $21,90 \pm 2,30$ & 26,65 & 19,34 & $0,04^{*}$ \\
\hline
\end{tabular}

*Valor $\mathrm{p}<0,05$.

\section{DISCUSIÓN}

Las relaciones morfológicas de los huesos y las suturas del paladar son fundamentales al considerar la ampliación multidireccional palatina de acuerdo al crecimiento cráneofacial. Tanto los huesos del paladar, como la red sutural que los interconecta se desarrollan en la etapa intrauterina (Cantin et al., 2011), y se va sinostosando paulatinamente. Pudimos observar que en los recién nacidos, todas las suturas del paladar están abiertas, observando grados de sinostosis en infantes que aumentan en los niños, junto al crecimiento longitudinal y transversal significativo de todos los grupos suturales.

El análisis morfométrico de las suturas palatinas suministra información acerca de las dependencias anatómicas que resultan de los principios del desarrollo craneofacial. Las suturas palatinas podrían ser consideradas como una piedra angular en la coordinación adecuada del crecimiento de todo el cráneo, y reflejan el modo de interacción entre los huesos palatinos y el maxilar durante su crecimiento gra- cias a su disposición en dos sistemas, sagital y transversal (Skrzat et al.). Estos se disponen en ángulos rectos entre sí, lo que permite un crecimiento bidireccional del paladar. Las SPPMX, SPMA y SPMP pertenecen al sistema sutural sagital y permiten el crecimiento transversal del paladar, mientras que la SPTA y SPTP facilitan el crecimiento longitudinal de éste (Skrzat et al.). Esto concuerda con las observaciones de Rojvachiranonda et al., quienes disecaron 8 recien nacidos entre las 27 semanas y el término de gestación y lograron separar la SPMA, SMPP y SPTP en todas las muestras, demostrando la movilidad de los huesos palatinos.

La región anterior del paladar óseo en adultos es la unión permanente entre el paladar primario y secundario, representada por la fosa incisiva (D'Souza et al., 2012), pero en RN se observa las uniones suturales de estos, las cuales se van obliterando durante la primera infancia (N'Guyen $e t$ $a l$.), y nuestros resultados confirman al momento del nacimiento no muestran ningun grado de obliteración, mientras 
que la edad de obliteración de la sutura palatina mediana fue variable, comienzando en la parte anterior, también con menor obliteración en las primeras etapas. Según Skrzat et al., en jóvenes el aumento longitud del paladar es predominanate, y se observa a partir de un incremento en la dimensión antero-posterior de la SPTP. Nuestros resultados coinciden con estas observaciones, ya que observamos un crecimiento longitudinal constante del paladar en RN, infantes y niños, mostrando diferencias significativas en todas las medidas; sin embargo es interesante observar que el crecimiento transvesal no tiene esta constancia, ya que este muestra una tasa elevada entre RN e infantes, pero disminuye en los niños. Esto significa que existen diferentes potenciales de crecimiento sutural, siendo el longitudinal mantenido y el transversal temporal, mostrando un peak dentro de los 2 primeros años de vida. Estas observaciones podrían ayudar a explicar la alta prevalencia de alteraciones de crecimiento de tipo transversal en los niños.

La comparación métrica entre la longitud de las suturas palatinas de las en paladares de sexo masculino y femenino no reveló dimorfismo sexual significativo, aunque existen diferentes tendencias entre los sexos y la correlación de longitudes especialmente en las suturas posteriores del paladar, lo cual puede ser el resultado de un patrón diferencial de crecimiento. Nuestros resultados coinciden con las observaciones de Skrzat et al., quienes reportaron para la SPMP en hombres una Media de 14,3 (DE 2,39) mm, y en mujeres Media de 14,1 (DE 2,110) mm, para SPMA en hombres una Media de 28 (DE 3,19) mm y mujeres de 25,8 (DE 2,55) mm, para SPTP en hombres una Media de 32,5 (DE 2,84) mm y mujeres de 32,1 (DE 2,61) $\mathrm{mm}$.

La longitud del sistema sutural sagital del paladar fue casi el doble que el transversal, además los procesos palatinos del maxilar constituyen aproximadamente el $65 \%$ del paladar óseo, mientras que las láminas horizontales del hueso palatino constituyen alrededor del $35 \%$. Sobre la base de estas consideraciones podemos inferir que el tamaño de los huesos palatinos, delimitado por la longitud de la sutura interpalatina, tiene un efecto significativo sobre las dimensiones longitudinales del paladar óseo, tanto en hombres como mujeres. Los resultados de estas observaciones y análisis métricos de las suturas palatinas pueden conducir a un nuevo concepto para la investigación anatómica sobre la coordinación del crecimiento cráneofacial, el que puede ser controlado por diferentes factores como la edad o el origen étnico (Ferrario et al.; Skrzat et al.).

La distracción osteogénica (DO) es una técnica en la cual el hueso se alarga paulatinamente bajo tensión después de una osteotomía. La aplicación de la DO a los trastornos craneofaciales se está investigando activamente, donde se ha intentado el la expansión de sutura en el paladar duro para lograr una elongación, y el cierre de fisura alveolar (Tibesar et al., 2005). En las deficiencias transversales maxilares, para realizar la expansión del paladar, las suturas deben estar abierta, y ésto sólo es posible según N'Guyen et al. hasta 13 años, donde la sutura palatina mesiana alcanza una longitud promedio de 25,09 $\pm 3,18 \mathrm{~mm}$. En los adultos, cuando la expansión palatina es necesaria, se requiere una separación quirúrgica previo al tratamiento de expansión por ortodoncia o expansión maxilar transversal.

Según Kumar et al. (2011) durante la infancia, la permeabilidad de las suturas es vital para expansion maxilar rápida (EMR), y es importante saber cuándo se cierra una sutura por sinostosis, donde el mayor grado de obliteración se produce desde posterior a anterior. Tratamientos como ERM se realizan a través de la sutura media palatina, donde la fuerza se transfiere a la sutura palatina, lo que resulta en la apertura sutural. Sin embargo algunos estudios informan efectos secundarios tales como los microtraumatismos de la sutura palatina mediana y recidivas conforme aumenta la edad del paciente, ya que las suturas se vuelven más interdigitadas, sobre todo después de la pubertad, donde se requiere una fuerza mayor para abrir las suturas (Huynh et al., 2009). Por esto, posiblemente la intervención en edaddes tempranas mejoren los pronósticos y recidivas; esto fue sugerido por Lui \& Song (2000), quienes experimentaron con un distractor posterior en cinco recién nacidos con fisura palatina logrando cierre completo.

Este enfoque temprano de intervención podría ayudar a superar las deficiencias y limitaciones del actual manejo quirúrgico de paladar hendido, ya que las técnicas contemporáneas con frecuencia dejan áreas del hueso palatino expuesto lo que genera contracción de la cicatriz, y contribuye a un deterioro posterior en el crecimiento del tercio medio facial, produciendo un acortamiento del paladar blando e insuficiencia velofaríngea posterior. Además, la tensión colocada en los colgajos mucosos palatales en el momento de la reparación quirúrgica en combinación con la falta de cualquier reparación ósea aumenta el riesgo de dehiscencia de la herida y la fístula oronasal. Estas complicaciones suelen requerir procedimientos más invasivos en edades más avanzadas de los niños (Tibesar et al.).

Algunos autores (Liu et al., 2005; Swennen et al., 2002) han planteado realizar una DO de suturas sin osteotomía para cerrar un paladar hendido mediante el avance combinado por alargamiento del paladar óseo posterior y anterior. Esto tendría varias ventajas, como la no morbilidad de donantes, aumento simultáneo en el hueso y 
tejido blando que lo cubre, de alta calidad la formación de hueso nuevo, y menos disección quirúrgica. La aplicación para el paladar se ha experimentado recientemente en animales con la esperanza de que la incompetencia velofaríngea y las condiciones de fisuras puedan ser manejadas de una nueva manera menos invasiva (Carls, 1997a, 1997b; Liu et al., 2000; Rojvachiranonda et al.). Tibesar et al. evaluaron la utilidad de DO para el cierre del paladar hendido utilizando modelos animales caninos adultos, con una distracción de $1 \mathrm{~mm} /$ día más un periódo de consolidación, logrando el cierre completo de los huesos del paladar en el $62,5 \%$ de los casos, concluyendo que la aplicación de este tipo de técnicas es un modelo seguro y bien tolerado, y este tratamiento innovador debería utilizarce para el tratamiento de niños que nacen con paladar hendido. Liu et al., también realizaron DO sutural en perros con paladar hendido, sin una osteotomía junto con un dispositivo de distracción, siendo capaces de avanzar simultáneamente en el maxilar y alargar el paladar caudalmente. Ambos procedimientos son posibles sin una osteotomía, ya que se utiliza tanto las suturas transversales y sagitales del paladar.
$\mathrm{Al}$ estimar el tiempo de apertura sutural del paladar en recién nacidos, infantes y niños, las reparaciones de defectos se pueden realizar en etapas tempranas de la vida, resultando en un menor número de problemas de alimentación y una mejor evolución del habla (Rojvachiranonda $e t$ al., 2003), y evitar las compliocaciones derivadas de la mayor rigidez del esqueleto facial con la edad, que restringe los movimientos óseos (Kumar et al.). Además, las cirugías ortognáticas convencionales no se realizan hasta la adolescencia, sin embargo, esto tiene un importante efecto negativo en el desarrollo psíquico del paciente (Saltaji et al., 2012).

El conocimiento del crecimiento del paladar y sus suturas podrían permitir el desarrollo de nuevos enfoque quirúrgicos basados en DO para el tratamiento de problemas relacionados con defectos palatinos transversales y longitudinales, así como para el tratamiento de niños con fisura palatina y defectos relacionados, por lo que es necesario mayores estudios in vivo para estimar su utilidad y si es superior a las técnicas convencionales.

CANTIN, M.; OLATE, S.; FONSEA, C.; INZUNZA, O.; CONTRERAS, F. \& SALGADO, G. Morphometrical study of human palatine sutures in newborns, infants and children for distraction osteogenesis treatment purposes. Int. Int. J. Morphol., 31(3):11301136, 2013.

SUMMARY: Structural and dimensional knowledge of palatal sutures are necessary for early treatment of deficiencies by transverse and longitudinal expansion techniques or distraction osteogenesis (DO). The aim was to study the status and dimensional or morphological changes of palatal sutures from birth to childhood. Forty one bony palates of both sexes, between 0 and 13 years and grouped in newborns $(\mathrm{NB} / \mathrm{n}=17)$, infants $(\mathrm{IN} / \mathrm{n}=12)$ and children $(\mathrm{CH} / \mathrm{n}=12)$ were analyzed. All palates were photographed and craniometrics points were scored to determine the longitudinal and transverse dimensions of the palatal sutures: premaxillary (PMX), anterior midpalatal suture (AMPS), posterior midpalatal suture (PMPS), anterior transverse palatal suture (ATPS) and posterior transverse palatal suture (PTPS). In addition, we evaluated the sutural and sexual dimorphism. The results were subjected to tests OneWay - ANOVA and Bonferroni t-test. AMPS, PMPS and PTPS no showed synostosis. PMX was observed partially sinostosed in 5.9\% of NB and $16.7 \%$ of $\mathrm{CH}$, and ATPS in $8.3 \%$ of IN and $41.7 \%$ of $\mathrm{CH}$. When comparing the sutural length between groups, the differences were significant in all cases $(\mathrm{p}<0.001)$. There were no differences in transverse sutures between IN and $\mathrm{CH}$ groups $(\mathrm{p}=0.32)$. Sexual dimorphism was observed between AMPS and PTPS groups. The palatal suture system plays an important role during growth by sagittal and transverse available, allowing bidirectional growth of the palate. Longitudinal growth is constant, while the cross shows a peak until the first 2 years of life and then decreases, suggesting the existence of different potentials growth. These observations may explain the high prevalence of abnormal transverse growth in children. These data are relevant to the appropriate treatment by expansion or DO in palate compression cases, velopharyngeal incompetence or cleft palate.

KEY WORDS: Palatine sutures; Hard palate; Palate bone; Growth; Bone distraction; Distraction osteogenesis.

\section{REFERENCIAS BIBLIOGRÁFICAS}

Cantín, M.; Fernández, R. F. \& Rojas, M. A proposed explanation for the development of the torus palatinus. Clin. Anat., 24(6):789-90, 2011.

Carls, F. R.; Jackson, I. T. \& Topf, J. S. Distraction osteogenesis for lengthening of the hard palate: Part I. A possible new treatment concept for velopharyngeal incompetence. Experi- mental study in dogs. Plast. Reconstr. Surg., 100(7):1635-47, 1997a.

Carls, F. R.; Schüpbach, P.; Sailer, H. F. \& Jackson, I. T. Distraction osteogenesis for lengthening of the hard palate: Part II. Histological study of the hard and soft palate after distraction. Plast. Reconstr. Surg., 100(7):1648-54, 1997b. 
D’Souza, A. S.; Mamatha, H. \& Jyothi, N. Morphometric analysis of hard palate in south Indian skulls. Biomed. Res., 23(2):173$5,2012$.

Ferrario, V. F.; Sforza, C.; Colombo, A.; Tartaglia, G. M.; Carvajal, R. \& Palomino, H. The effect of ethnicity and age on palatal size and shape: a study in a northern Chilean healthy population. Int. J. Adult Orthodon. Orthognath. Surg., 15(3):233-40, 2000

Huynh, T.; Kennedy, D. B.; Joondeph, D. R. \& Bollen, A. M. Treatment response and stability of slow maxillary expansion using Haas, hyrax, and quad-helix appliances: a retrospective study. Am. J. Orthod. Dentofacial Orthop., 136(3):331-9, 2009.

Kumar, S. A.; Gurunathan, D.; Muruganandham \& Sharma, S. Rapid maxillary expansion: a unique treatment modality in dentistry. J. Clin. Diagn. Res., 5(4):906-11, 2011.

Lathan, R. A. Maxillary development and growth: the septopremaxillary ligament. J. Anat., 107(3):471-8, 1971.

Liu, C. M.; Huang, X. M.; Hou, M.; Liang, L. M.; Xiao, M. \& Zhang, H. Z. Trans-sutural distraction osteogenesis for early correction of midfacial hypoplasia in children: a primary clinical report. Zhonghua Zheng Xing Wai Ke Za Zhi, 21(2):903, 2005 .

Liu, C.; Song, R. \& Song, Y. Sutural expansion osteogenesis for management of the bony-tissue defect in cleft palate repair: experimental studies in dogs. Plast. Reconstr. Surg., 105(6):2012-27, 2000.

Lui, C. \& Song, R. Sutural distraction osteogenesis for cleft palate repair. Presented at the 3rd Asian PacificAssociationConference, Shanghai, China, October, 2000.

Moreira, R. S.; Sgrott, E. A.; Seiji, F. \& Smith, R. L. Biometry of hard palate on digital photographs: A methodology for quantitative measurements. Int. J. Morphol., 24(1):19-23, 2006.

N'Guyen, T.; Gorse, F. C. \& Vacher, C. Anatomical modifications of the mid palatal suture during ageing: a radiographic study. Surg. Radiol. Anat., 29(3):253-9, 2007.

Rojvachiranonda, N.; Tansatit, T.; Siriwan, P. \& Mahatumarat, C. Normal palatal sutures in newborns and fetuses: a critical fact for successful palataldistraction. J. Craniofac. Surg., 14(4):457$61,2003$.

Saltaji, H.; Major, M. P.; Altalibi, M.; Youssef, M. \& Flores-Mir, C. Long-term skeletal stability after maxillary advancement with distraction osteogenesis in cleft lip and palate patients. Angle Orthod., 82(6):1115-22, 2012.

Silau, A. M.; Njio, B.; Solow, B. \& Kjaer, I. Prenatal sagittal growth of the osseous components of the human palate. J. Craniofac. Genet. Dev. Biol., 14(4):252-6, 1994.
Skrzat, J.; Holiat, D. \& Walocha, J. A morphometrical study of the human palatine sutures. Folia Morphol. (Warsz), 62(2):123-7, 2003.

Stuart, D. A. \& Wiltshire, W. A. Rapid palatal expansion in the young adult: time for a paradigm shift? J. Can. Dent. Assoc., 69(6):374-7, 2003.

Swennen, G.; Dempf, R. \& Schliephake, H. Cranio-facial distraction osteogenesis: a review of the literature. Part II: Experimental studies. Int. J. Oral Maxillofac. Surg., 31(2):12335, 2002.

Taub, P. J.; Bradley, J. P. \& Kawamoto, H. K. Closure of an oronasal fistula in an irradiated palate by tissue and bone distraction osteogenesis. J. Craniofac. Surg., 12(5):495-500, 2001.

Tibesar, R. J.; Moore, E. J. \& Bite, U. Distraction osteogenesis for cleft palate closure in a canine model. Arch. Facial Plast. Surg., 7(6):398-404, 2005

\section{Dirección para Correspondencia: \\ Mario Cantín \\ Departmento de Odontología Integral \\ Facultad de Odontología \\ Universidad de La Frontera \\ Manuel Montt 112, Temuco \\ CHILE}

Telefono + 560452325574

Email: mario.cantin@ufrontera.cl

Recibido: 04-03-2013

Aceptado: 16-06-2013 